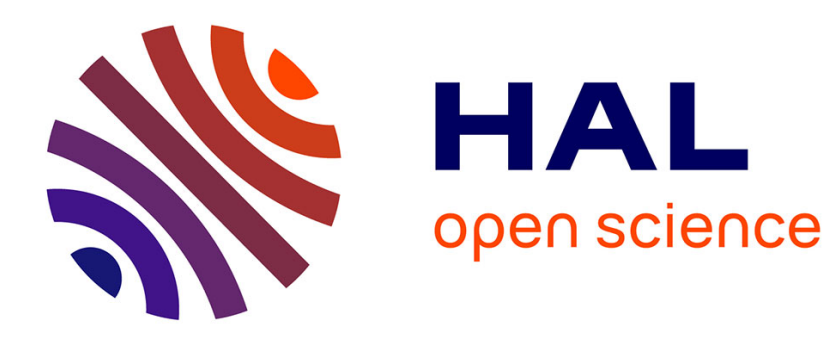

\title{
La notion actuelle de durée du travail peut-elle résister au coronavirus?
}

Jacques Barthelemy, Gilbert Cette

\section{To cite this version:}

Jacques Barthelemy, Gilbert Cette. La notion actuelle de durée du travail peut-elle résister au coronavirus ?. Regards, 2020, 57 (1), pp.13. 10.3917/regar.057.0013 . hal-03140469

\section{HAL Id: hal-03140469 \\ https://hal-amu.archives-ouvertes.fr/hal-03140469}

Submitted on 23 Feb 2021

HAL is a multi-disciplinary open access archive for the deposit and dissemination of scientific research documents, whether they are published or not. The documents may come from teaching and research institutions in France or abroad, or from public or private research centers.
L'archive ouverte pluridisciplinaire HAL, est destinée au dépôt et à la diffusion de documents scientifiques de niveau recherche, publiés ou non, émanant des établissements d'enseignement et de recherche français ou étrangers, des laboratoires publics ou privés. 


\title{
La notion actuelle de durée du travail peut- elle résister au coronavirus?
}

\author{
Par Jacques Barthélémy, Avocat conseil en droit social (honoraire) \\ et Gilbert Cette, Professeur associé à l'Université d' Aix-Marseille
}

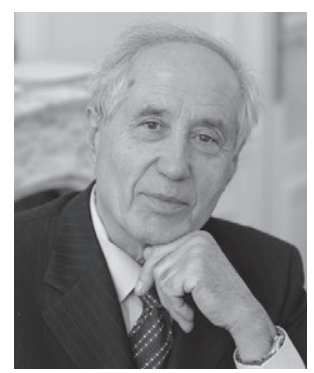

Jacques Barthélémy est avocat conseil en droit social, aujourd'hui honoraire, ancien professeur associé à la faculté de droit de Montpellier et fondateur (en 1965) du cabinet éponyme composé de plus de 130 avocats et présent dans 18 bureaux en France. Il est Chevalier dans l'Ordre national des Palmes académiques et Officier dans celui de la Légion d'honneur. Il est également ancien membre du Conseil économique et social, ancien membre du Conseil national des barreaux, ancien Président de l'Association des Avocats Conseils d'Entreprise et ancien Vice-président de l'Union nationale des professions libérales. Il est l'auteur de nombreux articles concernant le droit du travail et celui de la protection sociale, notamment complémentaire, et de plusieurs ouvrages dont Droit social, technique d'organisation de l'entreprise (2003 réédité en 2015 - éditions Lamy) et, en tandem avec Gilbert Cette, Réformer le droit du travail (2015) et Travailler au XXIe siècle, (2017), les deux parus aux éditions Odile Jacob.

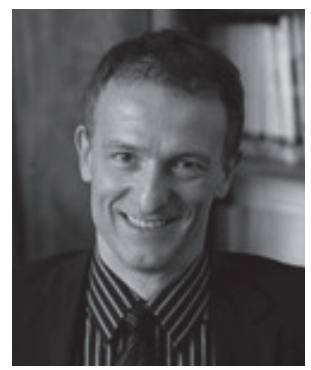

Gilbert Cette est professeur d'économie associé à l'Université d'AixMarseille. Il a obtenu un doctorat en économie à l'Université Paris 1. Il est Chevalier dans l'Ordre national de la Légion d'honneur. Il a été membre du Conseil d'Analyse Économique et du Groupe d'Experts sur le Smic qu'il préside actuellement. Il a présidé en 2012-2013 l'AFSE (Association Française de Science Économique). Gilbert Cette a effectué des recherches empiriques axées sur la croissance, la productivité, les innovations, l'économie du travail et les réformes structurelles et a publié dans de nombreuses revues comme The American Economic Review, The Journal of the European Economic Association, The Review of Economics and Statistics, The European Economic Review, The Review of Income and Wealth... Il a également publié plusieurs ouvrages, dont les derniers sont Le bel avenir de la croissance, avec Antonin Bergeaud et Rémy Lecat, Éditions Odile Jacob, 2018 et Travailler au XXle siècle, avec Jacques Barthelemy, Éditions Odile Jacob, 2017. 

e droit du travail a été façonné par la civilisation de l'usine et les modes hiérarchiques d'organisation du travail la caractérisant. Les mutations profondes nées des TIC s'amplifient et vont faire dépasser le droit du travail par celui de l'activité professionnelle dans la civilisation du savoir'. Ces mutations doivent favoriser l'épanouissement des droits fondamentaux dont les libertés, la dignité, la solidarité, la santé, la sécurité, la libre circulation des travailleurs.

Les obstacles à cette évolution sont d'ordre sociologique tant est grande dans les esprits la prééminence du contrat de travail... malgré sa définition par référence à un état de subordination. Qu'il faille inventer les instruments permettant de protéger la partie faible du contrat est une chose ; faire de la subordination un état privilégié en est une autre.

La situation que nous vivons du fait du coronavirus fera évoluer les mentalités. En particulier, le confinement a suscité de profondes mutations dans l'organisation du travail. Télétravail et travail à domicile deviendront d'autant plus habituels qu'ils sont porteurs de meilleures conditions de travail et de libertés pour le travailleur, de coûts moindres et de flexibilité pour l'entreprise. La flexibilité est porteuse pour le travailleur d'un plus grand confort dans la conciliation entre vies personnelle et professionnelle. Pour l'entreprise, l'abaissement des coûts et l'élévation de la productivité du travailleur peuvent venir, outre d'une plus grande satisfaction de ce dernier, de moindres temps de transport et d'une diminution des surfaces des locaux de l'entreprise.

Un domaine illustre particulièrement cette question : celui de la durée du travail. Nous abordons cette question successivement sous un angle quantitatif (I) puis qualitatif (II) avant d'évoquer d'autres dimensions en lien avec l'amélioration du bien commun qui peut être associé à ces mutations (III), puis de conclure par quelques remarques à caractère prospectif.

\section{I- LA DIMENSION QUANTITATIVE}

En droit français, la durée effective du travail est identifiée par le temps durant lequel le salarié est à disposition de l'employeur et se conforme à ses directives sans pouvoir vaquer à des occupations personnelles. On voit, dans cette définition, la filiation aux organisations hiérarchiques de travail. De ce fait, elle est de plus en plus inadaptée à de nombreuses fonctions et à de multiples activités. La jurisprudence tente de concilier cette règle avec des situations de fait exigeant des adaptations. Le législateur a dû parfois intervenir, par exemple, sur la question des forfaits jours ${ }^{2}$ au vu de l'inadéquation de la technique du forfait de salaire pour un nombre d'heures... La population concernée est en effet caractérisée par un degré élevé d'autonomie rendant de fait, sinon de droit, impossible le contrôle des heures. D'où l'importance de s'y référer au moment d'évoquer les mutations du travail liées au choc du coronavirus.

1 Voir à ce sujet Jacques Barthelemy et Gilbert Cette, 2017, op. cit.

2 Le recours au forfait jours est loin d'être une pratique anecdotique. Selon la Dares, près de $15 \%$ des salariés du secteur privé travaillaient en forfait jours en 2014, cette proportion étant de près de $50 \%$ pour les cadres (voir Dares, « Les salariés au forfait annuel en jours », Dares Analyse, 2015-048, juillet 2015, rédigé par Claire Letroublon). Le recours au forfait jours a d'ailleurs été récemment facilité par les ordonnances Travail en 2017 confirmées par la loi Pénicaud en mars 2018 : désormais, le forfait jours peut être adopté dans l'entreprise via un accord de performance collective. 
Dans le contexte du choc lié à la Covid-19 et du confinement, le Gouvernement s'est rapidement montré conscient des difficultés posées par un droit de la durée du travail conçu pour les modes hiérarchiques d'organisation du travail. En témoigne le contenu de l'ordonnance du 25 mars portant mesures d'urgence en matière de congés payés, de durée du travail et de jours de repos.

Le droit communautaire avait une définition similaire à celle du droit français mais des directives de 1993 puis de 2004 ont introduit un élément d'autant plus important qu'il permet de se référer à des droits fondamentaux, ceux à la santé et à la sécurité en particulier. À partir de cette finalité, la durée effective ne peut que gagner à être appréhendée par opposition à celle de repos. Ceci permet en effet de tenir compte plus aisément de spécificités tenant au degré d'autonomie du travailleur, du lieu du travail ou de la nature des tâches. On doit aussi tirer de ce que cette définition est en lien avec le droit à la santé, droit fondamental qu'elle peut être appliquée au travailleur non salarié, par exemple en cas de dépendance économique, ce qui peut contribuer au dépassement du droit du travail par celui de l'activité professionnelle. Un autre avantage est de prendre en compte pour l'évaluation du temps de travail tous les repos, journaliers et hebdomadaires certes, mais aussi annuels, c'est à dire les congés.

Avant le choc du coronavirus, le télétravail était une pratique peu répandue. Selon la Dares, en 2017, seuls $3 \%$ des salariés le pratiquaient au moins un jour par semaine 3 . Ce type de travail a connu une explosion durant la période de confinement. La Dares a évalué à cette occasion que près de 4 emplois sur 10 seraient aujourd'hui, dans le secteur privé, compatibles avec le télétravail ${ }^{4}$. Ce chiffrage qui concerne la France est assez proche de chiffrages concernant d'autres pays. Aux États-Unis, Dingel et Neiman $(2020)^{5}$ évaluent cette proportion à $34 \%$ en moyenne. Ils indiquent qu'elle connait d'importantes différences entre zones métropolitaines (de $26 \%$ pour la plus basse à $48 \%$ pour la plus élevée), entre secteurs d'activités (de $0 \%$ dans la construction et la restauration à $97 \%$ dans l'expertise juridique) et selon les professions (de $3 \%$ pour les employés des services alimentaires à $77 \%$ pour les experts en services scientifiques et techniques).

Dans de nombreuses situations comme celles favorisant le forfait jours ou le télétravail, la mesure quantitative du temps de travail laisse de fait la place à une appréciation de la charge de travail du salarié et au-delà du travailleur, les non-salariés indépendants étant aussi concernés. Cette approche passe tout d'abord par le droit à la déconnexion garantissant au travailleur que, hors circonstances exceptionnelles dont la nature peut d'ailleurs être prévue, il ne recevra pas d'instructions et ne sera pas tenu de répondre à toute injonction en dehors de certaines plages horaires ou de certains jours. Depuis la loi El Kohmri de 2016, ce droit à la déconnexion fait partie des sujets à aborder lors de la négociation annuelle obligatoire (NAO). Il peut aussi être défini par une charte élaborée après avis des institutions représentatives du personnel (IRP) mais aussi par accord collectif.

3 Voir Dares, «Quels sont les salariés concernés par le télétravail ? », Dares Analyses, 2019-051, novembre 2019, rédigé par Sébastien Hallépée et Amélie Mauroux.

4 Voir Dares, «Coronavirus et monde du travail », communiqué de presse du 15 mars 2020.

5 Jonathan I. Dingel et Brent Neiman, « How Many Jobs Can be Done at Home? », NBER, Working paper, N²6948, April 2020. 
Cette approche implique néanmoins une appréciation de la charge de travail confiée au travailleur. Une même charge de travail peut être adaptée au respect des limites du temps de travail effectif pour certains travailleurs, mais non pour d'autres. Cette nouvelle approche nécessite donc une appréciation individuelle des capacités des travailleurs, notamment pour éviter des dégradations de la santé, ainsi que des méthodes de management autrement plus sophistiquées que le contrôle du simple respect d'horaires collectifs. Il y a bien sûr ici le risque de contentieux, mais ces derniers sont des options bien évidemment incertaines tant pour le travailleur que l'employeur, les deux parties devant apporter au juge des éléments de fait permettant d'apprécier la lourdeur effective de la charge de travail. Les mutations en cours dynamisées par le choc lié à la Covid-19 vont donc appeler de profondes transformations des modes de management, ce qui signifie que certaines entreprises s'y adapteront mieux que d'autres, et pourront en tirer des avantages au plan concurrentiel, donc économique.

\section{II - LA DIMENSION QUALITATIVE}

Les aspects qualitatifs du temps de travail sont une dimension essentielle de la problématique ouverte par le sujet de cet article. La possibilité de sortir de l'horaire collectif - et souvent uniforme - est un progrès au plan des libertés... si le travailleur y est associé. Les attentes des salariés en ce domaine sont très variées et, en conséquence, la meilleure conciliation entre vies personnelle et professionnelle pourra prendre des formes très spécifiques. La garde de jeunes enfants, les soins à un proche âgé ou handicapé, l'engagement dans des activités sportives, artistiques ou associatives sont des occupations dont la meilleure articulation avec le travail appelle une organisation quasi individualisée de l'imbrication des différents temps de vie. Forfait jours et télétravail peuvent faciliter cette optimisation individuelle. Des enquêtes d'opinion montrent par exemple que les travailleurs en forfait jours les plus satisfaits de ce changement sont d'un côté les cadres, du fait du flou des frontières entre certaines activités relevant ou non du travail, de l'autre les parents, les femmes surtout, de jeunes enfants ${ }^{6}$.

Cela signifie que les modes d'organisation en télétravail ou forfaits jours doivent faciliter l'articulation entre vies personnelle et professionnelle. Une tension est inévitable, dans de nombreux cas, entre une telle individualisation et le suivi hiérarchique de la réalisation de la charge de travail, mais aussi avec l'effectivité d'un collectif de travail souvent indispensable pour la circulation de l'information et la recherche de gains de productivité. Ici encore, les modes de management nécessitent des évolutions fondamentales allant de pair avec un exercice totalement transformé de l'autorité hiérarchique. Dans de nombreux travaux, Bloom et Van Reenen (voir par exemple 20107) ont montré que les modes de management ont un impact important sur l'efficacité productive des firmes, en particulier lorsque celles-ci savent associer des incitations notamment financières à des objectifs clairement définis et à un suivi régulier. Ils montrent que, au sein d'un échantillon d'une vingtaine de pays, la France serait à cet

6 Voir à ce sujet Gilbert Cette, Nicolas Dromel et Dominique Méda, « Les déterminants du jugement des salariés sur la RTT », Economie et Statistique, n³76-377, 2004, pp. 117-151.

7 Nicholas Bloom et John Van Reenen, "Why do management practices differ across firms and countries », Journal of economic Perspectives, Vol. 24, N 1, Winter 2010, pp. 203-224. 
égard dans une situation médiane de qualité managériale, les pays dans lesquels cette qualité serait globalement la meilleure étant les États-Unis, la Suède, le Japon et le Canada.

L'autonomie du travailleur dans l'organisation de l'articulation de ses temps de vie gagne à être organisée par accord collectif. La promotion croissante du rôle du dialogue social dans les changements réglementaires des dernières décennies, avec l'accélération produite par les ordonnances Travail de septembre 2017 et la loi Pénicaud de mars 2018, augmente largement cette possibilité. À cet égard, il est important de souligner que c'est en matière d'aménagement des temps de travail qu'a été mise en œuvre en premier la technique de dérogation, dès l'ordonnance de janvier 1982 abaissant à 39 heures la durée hebdomadaire légale du travail et les loi Auroux la même année. La technique dérogatoire est optimisée aujourd'hui par le principe de supplétivité qui élargit son champ aux rapports entre convention collective de branche et accord d'entreprise ${ }^{8}$. L'aménagement des temps de travail ne peut que s'amplifier encore du fait des TIC et de la digitalisation de l'économie, ainsi que de l'intérêt des entreprises pour le télétravail amplifié par les avantages aperçus dans la gestion du confinement lié au choc du coronavirus. La mise en œuvre de ces mutations gagnera à recourir au dialogue social, surtout à l'accord collectif, pour que les solutions concrètes soient à la fois adaptées au contexte et approuvées par les travailleurs. Le dialogue social peut être un outil de management et de transformations de l'entreprise tout en étant un droit fondamental protecteur du travailleur.

\section{III - AUTRES DIMENSIONS}

De telles mutations du travail vont cependant soulever un problème sérieux : celui des inégalités. En effet, ces nouvelles formes plus flexibles du travail concernent, certes pas uniquement mais surtout, les catégories professionnelles les plus élevées. Ces différences sont bien illustrées dans le recours au forfait jours ou le télétravail. Le forfait jours concerne plus particulièrement les cadres et, parmi les non-cadres, ceux qui exercent des fonctions d'encadrement ainsi que des fonctions technico-commerciales ${ }^{9}$. Les travailleurs concernés sont de ce fait en moyenne relativement plus diplômés que les autres. On retrouve ces mêmes caractéristiques concernant le télétravail10.

On ne peut se satisfaire pleinement de transformations, et considérer qu'elles élèvent le progrès social, si par ailleurs elles renforcent des inégalités au bénéfice des catégories les plus diplômées et les mieux rémunérées. Trois réponses semblent pouvoir être apportées à cette difficulté.

Une première réponse est que de nombreuses fonctions support qui ne comptent pas parmi les plus qualifiées peuvent aussi être éligibles à ces transformations du travail. On peut mentionner pour exemple les tâches de secrétariat, administratif ou de direction, qui sont souvent réalisables à distance. Dans son dénombrement des activités éligibles au télétravail, dans le contexte de la crise de la Covid-19, la Dares (2020, op. cit.) a inclus ce type de salariés. Elle apparait aussi dans l'évaluation

8 Voir sur ces aspects Jacques Barthelemy et Gilbert Cette, 2015, op. cit.

9 Voir à ce sujet Dares, 2015, op. cit.

10 Voir à ce sujet Dares, 2019, op. cit. 
proposée pour les États-Unis par Dingel et Neiman (2020, op. cit.). Cette réponse n'est évidemment que très partielle, car si l'augmentation du nombre des salariés concernés atténue l'inégalité initiale, celle-ci demeure cependant forte.

La deuxième réponse est celle de la mobilité professionnelle et sociale. Les moyens d'une telle mobilité sont surtout ceux émanant de la formation professionnelle. Les travailleurs aspirant à changer de profession doivent pouvoir exprimer cette ambition avec une réelle autonomie de décision. Tel est bien l'un des objectifs de la réforme de la formation professionnelle et de l'apprentissage portée par la loi du 5 septembre 2018 dont la mise en œuvre progressive est en cours. Un bilan des stratégies construites en la matière devra être tiré dans quelques années afin d'élaborer un arsenal réglementaire ou conventionnel ${ }^{11}$.

Une dernière réponse peut prendre la forme de contreparties financières, via des primes spécifiques, mais aussi de durées du travail plus courtes. Cette dernière forme de contreparties parait assez logique : les nouvelles formes de travail (forfait jours, télétravail...) permettent à ceux qui les pratiquent, par l'autonomie organisationnelle qu'elles ouvrent, une meilleure conciliation entre vies personnelle et professionnelle. Des temps de travail allégés permettent, de même, une meilleure conciliation entre ces différents temps de vie aux travailleurs dont les horaires et l'organisation du travail demeureront fortement contraints et prescrits. Ces contreparties mériteraient d'être définies par accord d'entreprise, qui devrait simultanément s'intéresser aux modalités définissant les formes et degrés de l'expression de l'autonomie des autres travailleurs.

\section{CONCLUSION}

Les modes exceptionnels d'organisation du travail imposés par le confinement et demain par la grave crise économique qui se dessine vont conduire à des modifications profondes des comportements prenant appui sur le fait que, avec de l'imagination, l'arsenal juridique peut favoriser l'épanouissement des droits de l'Homme en les conjuguant avec l'efficacité économique, qui est notamment au service de l'emploi.

Les développements ci-dessus peuvent être repris pour d'autres domaines touchant aux rapports de travail, au premier rang desquels le statut du travailleur, dès lors que la référence à la subordination juridique pour bénéficier de l'arsenal protecteur peut être utilement revisitée. La protection de la partie faible doit permettre, entre autres avantages, de remplacer les sanctions négatives de type disciplinaire par des sanctions positives plus à même de respecter la dignité de l'Homme et de créer du bien-être au travail, donc de favoriser l'efficacité économique.

Définir négativement le temps de travail par opposition à celui de repos et faciliter l'individualisation des horaires en déconnectant la rémunération du nombre d'heures peut contribuer à l'émergence d'un droit nouveau valorisant les droits fondamentaux de l'Homme sans altérer la finalité du travail pour lequel la rémunération est établie...

11 Ce thème de la mobilité professionnelle était celui du Séminaire Emploi qui s'est déroulé le 30 novembre 2018. Il y a été montré que cette mobilité est actuellement, et avant cette réforme, nettement plus faible que dans de nombreux pays développés, et que la moyenne de l'Union Européenne. Voir : https://www.tresor.economie. gouv.fr/Articles/8a0b2a5c-1e10-4e87-b479-a722551846f2/files/05b035dc-04ba-448c-980c-7cb7ff90762d. Or la mobilité des travailleurs est considérée par la Charte des droits sociaux de l'Union européenne comme un droit fondamental. 